Addendum

\title{
De Sousa Luis, J.A., et al. Synthesis of New Imidazolidin-2,4- dione and 2-Thioxo-imidazolidin-4-ones via C-Phenylglycine Derivatives. Molecules 2010, 15, 128-137
}

José Alixandre de Sousa Luis ${ }^{1,2}$, José Maria Barbosa Filho ${ }^{1}$, Bruno Freitas Lira ${ }^{1}$, Isac Almeida Medeiros ${ }^{1}$, Liana Clébia Soares Lima de Morais ${ }^{1}$, Raline Mendonça dos Anjos ${ }^{1}$, Alexsandro Fernandes dos Santos ${ }^{3}$, Cledualdo Soares de Oliveira ${ }^{3}$ and

Petrônio Filgueiras de Athayde-Filho ${ }^{1,3, *}$

1 Laboratório de Tecnologia Farmacêutica, Universidade Federal da Paraíba, João Pessoa - PB, CEP 58.051-970, Brazil

2 Centro de Educação e Saúde, Unidade Acadêmica de Saúde, Universidade Federal de Campina Grande, Cuité - PB, CEP 58.175-000, Brazil

3 Departamento de Química, Universidade Federal da Paraíba, Campus I, João Pessoa - PB, CEP 58059-900, Brazil

* Author to whom correspondence should be addressed; E-Mail: athayde-filho@quimica.ufpb.br.

Received: 12 February 2010 / Published: 22 March 2010

The authors wish to make the following correction to their paper [1], published recently in Molecules. The coauthor Raline Mendonça dos Anjos was omitted from the author list, which should read as follows:

José Alixandre de Sousa Luis ${ }^{1,2}$, José Maria Barbosa Filho ${ }^{1}$, Bruno Freitas Lira ${ }^{1}$, Isac Almeida Medeiros ${ }^{1}$, Liana Clébia Soares Lima de Morais ${ }^{1}$, Raline Mendonça dos Anjos ${ }^{1}$, Alexsandro Fernandes dos Santos ${ }^{3}$, Cledualdo Soares de Oliveira ${ }^{3}$ and Petrônio Filgueiras de Athayde-Filho ${ }^{1,3, *}$

\section{References}

1. De Sousa Luis, J.A.; Barbosa Filho, J.M.; Freitas Lira, B.; Almeida Medeiros, I.; Soares Lima de Morais, L.C.; dos Santos, A.F.; Soares de Oliveira, C.; de Athayde-Filho, P.F. Synthesis of New Imidazolidin-2,4-dione and 2-Thioxoimidazolidin-4-ones via C-Phenylglycine Derivatives. Molecules 2010, 15, 128-137. 
(C) 2010 by the authors; licensee Molecular Diversity Preservation International, Basel, Switzerland. This article is an open-access article distributed under the terms and conditions of the Creative Commons Attribution license (http://creativecommons.org/licenses/by/3.0/). 